\title{
The effect of elbow position on the handgrip strength test in children: validity and reliability of TKK 5101 and DynX dynamometers
}

\author{
Stefan Kolimechkov ${ }^{1 \mathrm{ABCD}}$, Jose Castro-Piñero ${ }^{2 \mathrm{CD}}$, Lubomir Petrov ${ }^{3 \mathrm{ACD}}$, Albena Alexandrova ${ }^{3 \mathrm{CD}}$ \\ ${ }^{1}$ STK SPORT, London, United Kingdom \\ ${ }^{2}$ University of Cadiz, Puerto Real, Spain \\ ${ }^{3}$ National Sports Academy, Sofia, Bulgaria
}

Authors' Contribution: A - Study design; B - Data collection; C - Statistical analysis; D - Manuscript; Preparation; E - Funds Collection.

\begin{abstract}
Purpose: $\quad$ One of the most widely applied methods to assess upper-body strength in children and adolescents is the handgrip strength test. While in adolescents it has been determined which elbow position, and which type of dynamometer are most appropriate for achieving the maximal grip strength, in children is still not clear. Therefore, the purpose of this study was to investigate whether elbow position and the type of dynamometer affect the handgrip strength in children.

Material: $\quad$ Grip strength was measured by TKK and DynX dynamometers, and their validity and reliability were also analysed. A total of 60 children, 6 to 11 years old, participated in this study, and performed the handgrip strength test with their elbow extended and flexed at $90^{\circ}$.

Results: $\quad$ While using the TKK dynamometer, grip strength was significantly higher when the test was performed with elbow extended, in contrast to those obtained with elbow flexed $(14.58 \pm 3.04 \mathrm{~kg}$ vs $12.97 \pm 2.99 \mathrm{~kg}$, $\mathrm{p}<0.001$ for right, and $14.25 \pm 3.05 \mathrm{~kg}$ vs $12.61 \pm 2.99 \mathrm{~kg}, \mathrm{p}<0.001$ for left hand). Likewise, when using the DynX dynamometer, the difference between the two elbow positions was smaller but still significant (13.84 $\pm 3.22 \mathrm{~kg}$ vs $13.35 \pm 3.01 \mathrm{~kg}, \mathrm{p}=0.035$ for right, and $13.35 \pm 2.95 \mathrm{~kg}$ vs $12.77 \pm 2.96 \mathrm{~kg}, \mathrm{p}=0.003$ for left hand).

Conclusions: $\quad$ Both dynamometers provided sufficient results in terms of their reliability and the TKK dynamometer showed to be more valid. Performing the handgrip strength test with elbow extended appears to be the most appropriate position in order to obtain maximal results in children.

Keywords: physical fitness, maximum grip performance, dynamometry, Bland and Altman.
\end{abstract}

\section{Introduction}

Muscular strength is the ability to perform activities which require a high level of muscular force and has been one of the main components for assessing health-related physical fitness [1]. Systematic reviews show that there is strong evidence that improvements in muscular strength from childhood to adolescence are inversely proportional to changes in overall adiposity later in life $[2,3]$. There is no single test for measuring muscle strength, because the maximum force which can be generated depends on several factors, such as the size and number of muscles involved, the proportion of muscle fibres in action, and the coordination of the muscle groups [4]. However, the most commonly performed test is the handgrip strength test $[1,4]$, which appears to be a widely-used method to measure upper body isometric strength in children and adolescents [5-9].

Handgrip strength has been shown to be influenced by different factors, such as gender, age, positions of the shoulder, elbow, forearm and wrist [10-12], posture $[13,14]$, as well as grip span and hand span [4, 15-18]. It is not clear which elbow position is most appropriate for achieving the maximal handgrip strength, owing to inconsistent findings [19-23]. While the American Society of Hand Therapists supports the $90^{\circ}$ flexed elbow

\footnotetext{
(c) Stefan Kolimechkov, Jose Castro-Piñero, Lubomir Petrov

Albena Alexandrova, 2020

doi:10.15561/26649837.2020.0504
}

position [24], the American Centers for Disease Control and Prevention (CDC) recommends that the test should be performed with a straight elbow [25]. Furthermore, other findings show that maximal handgrip strength was achieved with flexed elbow at $135^{\circ}[26]$. Most studies on the influence of elbow position with regards to the assessment of handgrip strength have been conducted with adults [21, 23] or elderly people [20]; however, studies on the effect of elbow position in youngsters are scarce. For instance, it was reported that the most appropriate protocol for assessing handgrip strength, in adolescents between the ages of 12 and 16, is with elbow in full extension [27], but in children this has not been investigated yet.

Studies on handgrip strength have been conducted by using different hand dynamometers, such as: TKK digital dynamometer, Jamar hydraulic dynamometer and DynX electronic dynamometer. The validity and reliability of these common dynamometers were reported in several studies [28, 29], but they were mainly conducted by using correlation coefficients, which is not considered as the most appropriate method for that purpose [30, 31]. An alternative approach, based on graphical techniques and simple calculations for assessing agreement between two different methods, which was provided by Bland and Altman [32], is commonly used for that purpose. Based on this approach, studies on test and retest were conducted mainly with the TKK dynamometer, which was shown 
to be reliable by producing a mean difference of less than $0.25 \mathrm{~kg}$ [33-36], that is lower than the precision of an average analog dynamometer (i.e. $0.5 \mathrm{~kg}$ ). However, differences amongst instruments (type of dynamometers) and duration between measures might have an effect on the precision of a dynamometer [35]. Furthermore, when different models of the TKK dynamometer were measured, the systematic error between different instruments of the same model and between different models (digital vs. analog) was reported to be between $0.3 \mathrm{~kg}$ and $0.6 \mathrm{~kg}$, and the systematic error between new and old dynamometers ranged from $0.8 \mathrm{~kg}$ to $1.1 \mathrm{~kg}$ [37] which can be quite significant. The TKK dynamometer showed the lowest systematic error and the highest criterion-related validity and reliability in comparison to the Jamar and DynX dynamometers, when tested vs known weights within the range of 5-65 $\mathrm{kg}$ [27]. However, the Bland and Altman plots in the same study suggest that these results might be different in the range of the children's grip strength (5$30 \mathrm{~kg}$ ) for the DynX dynamometer. Although, the TKK dynamometer provided the highest validity and reliability (vs other dynamometers, such as Jamar), further research involving different types of dynamometers (e.g. Jamar, DynX), especially focused on the range of the children's handgrip strength (5-30 kg), are needed in order to confirm these findings [37].

Therefore, the purpose of our study was to investigate whether elbow position affects the handgrip strength in children ( $6-11$ years of age), and to analyze the validity and reliability of widely applied dynamometers, TKK and DynX vs known weights, within the range of the children's strength $(5-30 \mathrm{~kg})$.

\section{Material and methods}

In order to examine whether the elbow position affects the handgrip strength in children, grip strength measurements of two different elbow positions were compared (at full extension and flexed at $90^{\circ}$ ) by implementing two different hand dynamometers, TKK digital hand dynamometer and DynX electronic hand dynamometer. The Bland-Altman method was applied in order to analyze the validity and reliability of the TKK and DynX dynamometers vs known weights within the range of the children's handgrip strength $(5-30 \mathrm{~kg})$.

\section{Participants}

In this study a total of 60 children participated ( 30 boys and 30 girls) from 6 to 11 years of age. The research was carried out at St Edmund's Primary School in London, United Kingdom. Letters with information about the nature and the purpose of the study were given to the children and their parents/guardians. Written informed consent was obtained from the parents prior to testing. The protocols from this study were performed in accordance with the ethical standards established in the WMA Declaration of Helsinki (revised in Fortaleza, Brazil, in 2013).

\section{Procedures}

Handgrip strength was measured with two different dynamometers:

1. TKK digital hand dynamometer (TKK 5101 Grip-D, Takey, Tokyo, Japan), which has an adjustable grip span ranging from 3.5 to $7 \mathrm{~cm}$ with a precision of $0.1 \mathrm{~kg}[5]$.

2. DynX electronic hand dynamometer (MD System, Inc., Westerville, OH, USA), which has 3 grip
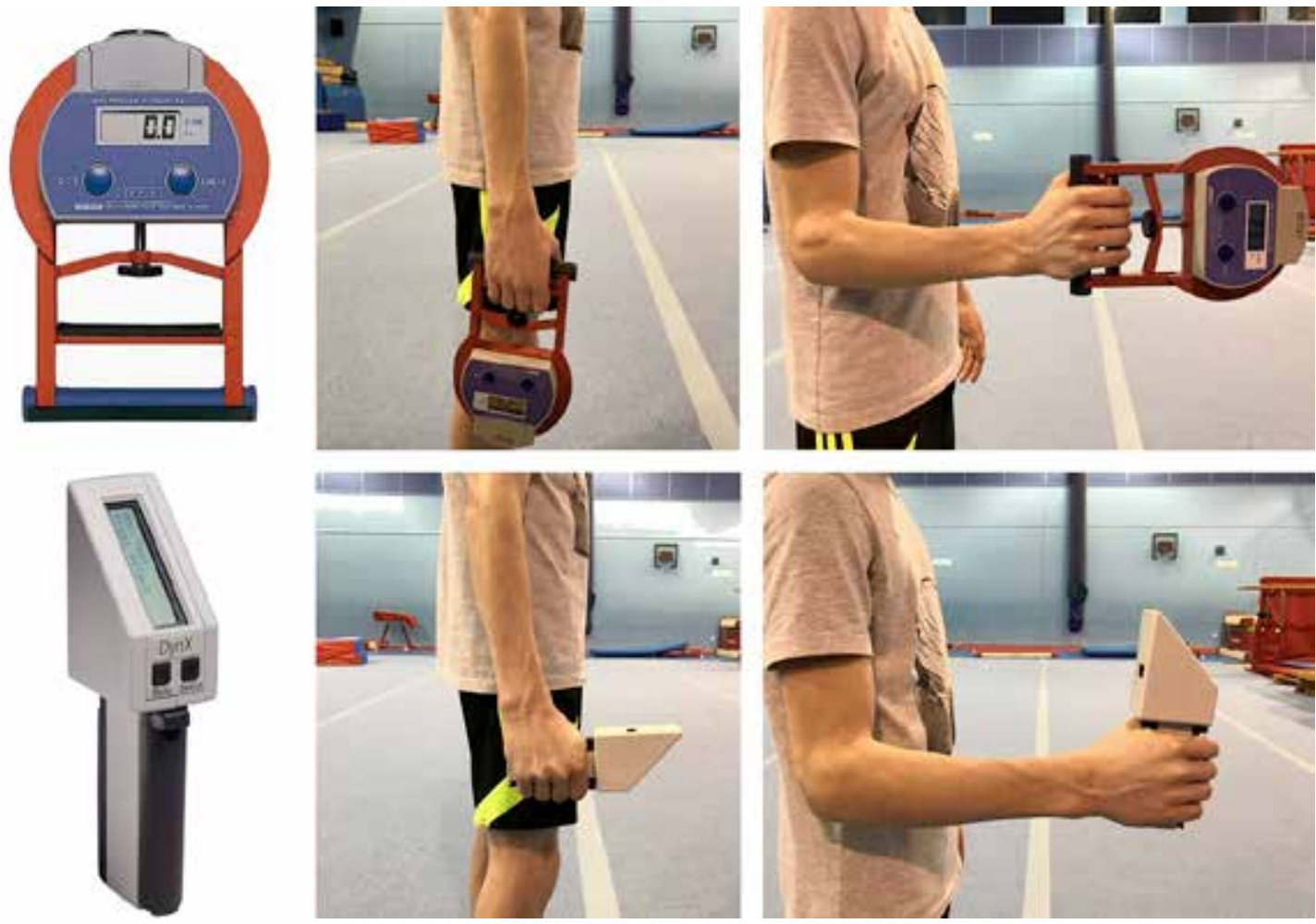

Fig. 1. Handgrip strength test with fully extended and flexed elbow, using TKK (above) and DynX (below) dynamometers 
positions $(4.75 \mathrm{~cm}, 6 \mathrm{~cm}$, and $7.25 \mathrm{~cm})$ with a precision of $0.1 \mathrm{~kg}$ [38].

The optimal grip span was determined for every child according to their hand size by employing the equations developed by Espana-Romero et al. [15], and the grip position of the TKK dynamometer was adjusted to each individual prior to testing. The second grip position (4.75 $\mathrm{cm})$ was used for the DynX dynamometer [28, 38].

Two different tests with each dynamometer were performed. The first test was with the elbow at full extension, and the second with flexed elbow at $90^{\circ}$. Standard procedures were followed for both tests [5, 27]. In brief, during the assessment with straight arm, children were asked to keep their elbow at full extension, the forearm in neutral position, with the wrist extended. When performing the test with bent elbow, the forearm was in neutral position and the elbow joint at $90^{\circ}$. Both tests were performed with feet shoulder-width apart in standing position, and children were given instructions not to touch any other part of the body with the dynamometer, except the hand being measured.

Each test was performed twice, with the left and right hand alternately. During the tests, the elbow position, the hand being tested, as well as the dynamometer were chosen randomly. Children were encouraged to squeeze gradually, continuously, and as hard as they can for at least two seconds, with a rest period of at least 60 seconds between tests and at least 5 min between dynamometers.

\section{Validity and reliability}

The comparison between dynamometers and known weights (validity) and repeated measurements (reliability) of the TKK and DynX dynamometers were analyzed by using known weights within the range of the children's grip strength $(5-30 \mathrm{~kg}$, every $5 \mathrm{~kg})$. The weights were verified by using an electronic scale with an accuracy of $0.05 \mathrm{~kg}$. The weights were held with a belt $(5 \mathrm{~cm}$ width and weight of $200 \pm 1 \mathrm{~g}$, which was taken into account), and they were measured twice in random order. In order to assess whether there was systemic bias, the deviation of the average differences of measurements from 0 was analyzed by one-sample t-test.

Statistical Analyses

The Kolmogorov-Smirnov test of normality was applied in order to examine the distributions of handgrip strength values. Paired t-test for dependent samples was used in order to compare the differences between the elbow positions (full extension vs flexed at $90^{\circ}$ ). Statistically significant differences between the average results were evaluated at $\mathrm{p}<0.05$, and all data in the text are presented as mean \pm SD. In addition, Cohen's effect size of the handgrip strength values with elbow at full extension vs flexed at $90^{\circ}$ were also calculated in order to present the magnitude of the effects. The following classification of the effect size was applied: $d(0.01)=$ very small, $\mathrm{d}(0.20)=$ small, $\mathrm{d}(0.50)=$ medium, $\mathrm{d}(0.80)$ $=$ large, $\mathrm{d}(1.20)=$ very large, and $\mathrm{d}(2.00)=$ huge $[39$, 40]. Percentile scores for the average handgrip strength of both hands were calculated by using the available gender- and age- specific interpolated European norms for children and adolescents [41]. All the analyses were performed for both boys and girls together because there was no interaction between gender and elbow position, and gender and type of dynamometer.

The Bland-Altman method was used to analyze the validity and reliability of the TKK and DynX dynamometers. This method is considered appropriate for studying the agreement of 2 measurements [32]. Moreover, the systematic bias, $95 \%$ confidence intervals of the bias, and the $95 \%$ limits of agreement (bias \pm 1.96 $\mathrm{SD}$ of the differences) were also computed.

\section{Results}

The Kolmogorov-Smirnov test showed normal distribution for all variables. The results from the handgrip strength test obtained with each dynamometer, and each hand and elbow position, are presented in Table 1. Handgrip strength was significantly higher when the test was performed with elbow extended, in contrast to the results obtained with flexed elbow at $90^{\circ}$, while using the TKK dynamometer $(14.58 \pm 3.04 \mathrm{~kg}$ vs $12.97 \pm 2.99$ $\mathrm{kg}, \mathrm{p}<0.001$ for right hand, and $14.25 \pm 3.05 \mathrm{~kg}$ vs 12.61 $\pm 2.99 \mathrm{~kg}, \mathrm{p}<0.001$ for left hand). Furthermore, Cohen's effect size values were 0.87 for right hand, and 0.91 for left hand. When using the DynX dynamometer, the difference between the two elbow positions was smaller, but still significant, in favour of the fully extended elbow position $(13.84 \pm 3.22 \mathrm{~kg}$ vs $13.35 \pm 3.01 \mathrm{~kg}, \mathrm{p}=0.035$ for right and, and $13.35 \pm 2.95 \mathrm{~kg}$ vs $12.77 \pm 2.96 \mathrm{~kg}, \mathrm{p}$ $=0.003$ for left hand), and the effect sizes were small to moderate $(\mathrm{d}=0.28$ and $\mathrm{d}=0.41$, respectively).

The data did not change much after adding up the results obtained with both hands (Figure 2). The children's grip strength was significantly higher when the test was performed with full extension, in contrast to bent elbow position using TKK dynamometer $(28.83 \pm 5.85 \mathrm{~kg}$ vs $25.58 \pm 5.69 \mathrm{~kg}, * \mathrm{p}<0.001)$, as well as using DynX

Table 1. Handgrip strength according to the type of dynamometer and hand and elbow position (at full extension and flexed at $\left.90^{\circ}\right)$ in children aged 6 to 11 years $(n=60)$, Mean \pm SD

\begin{tabular}{lllllll}
\hline Dynamometer & Hand & Extended & Flexed & Difference & $\begin{array}{l}\text { Percent } \\
\text { Difference }\end{array}$ & $\mathbf{p}$ \\
\hline \multirow{2}{*}{ TKK } & Right & $14.58 \pm 3.04$ & $12.97 \pm 2.99$ & $1.61 \pm 1.85$ & $11.02 \%$ & $<0.001$ \\
& Left & $14.25 \pm 3.05$ & $12.61 \pm 2.99$ & $1.64 \pm 1.81$ & $11.52 \%$ & $<0.001$ \\
DynX & Right & $13.84 \pm 3.22$ & $13.35 \pm 3.01$ & $0.49 \pm 1.77$ & $3.56 \%$ & 0.035 \\
& Left & $13.35 \pm 2.95$ & $12.77 \pm 2.96$ & $0.57 \pm 1.40$ & $4.28 \%$ & 0.003 \\
\hline
\end{tabular}


dynamometer $(27.19 \pm 5.86 \mathrm{~kg}$ vs $26.12 \pm 5.73 \mathrm{~kg}, * \mathrm{p}$ $<0.001)$. Additionally, significantly higher results were registered when using TKK dynamometer with full extension in comparison with DynX dynamometer with elbow flexed at $90^{\circ}, \dagger \mathrm{p}<0.001$ (Figure 2).

Percentile scores (PRs) of the average handgrip strength in both hands, based on the most recent norms in European children [41], were calculated from the values obtained by the TKK dynamometer with straight elbow position, because these results showed the highest values. The mean percentile score for all participants was $54.0 \pm$ 28.0, and ranged from 5.2 to 98.1 , which shows that the sample represents the whole range of possible handgrip values $(9.0-21.2 \mathrm{~kg})$ in children from 6 to 11 years of age.

The validity and reliability of the TKK and DynX dynamometers were analysed by using known weights within the range of the children's possible handgrip strength $(5-30 \mathrm{~kg})$. The criterion-related validity analyses showed a non-significant systematic bias of $-0.20 \mathrm{~kg}(\mathrm{p}>$ 0.05 ) for the TKK, and a significant systemic bias of -0.42 $\mathrm{kg}(\mathrm{p}<0.001)$ for the DynX dynamometer. The $95 \%$ limits of agreements were $0.62 \mathrm{~kg}$ for the TKK dynamometer, and $0.46 \mathrm{~kg}$ for the DynX dynamometer (Fig.3).

The reliability analyses revealed a non-significant systematic bias of $-0.07 \mathrm{~kg}$ in the TKK, and $0.10 \mathrm{~kg}$ in the DynX dynamometer ( $\mathrm{p}>0.05$ for both dynamometers), and the $95 \%$ limits of agreements were $0.27 \mathrm{~kg}$, and 0.25 kg, respectively (Fig.4).

\section{Discussion}

The handgrip strength test is part of many healthrelated physical fitness test batteries in children [5, 8, 42-
44], in addition to being widely used in experimental and epidemiological research $[7,45,46]$, as well as throughout a range of groups practising sports $[47,48]$. Therefore, it is of particular importance to standardize the procedure of this test in order to prevent measurement errors.

To the best of our knowledge there have not been any research focused on whether the elbow position affects handgrip strength in children between the ages of 6 and 11 when using TKK or DynX dynamometers. The results from this study, showing that handgrip strength is higher with full extension in comparison to bent elbow, are in agreement with a number of authors who investigated this issue in adolescents [27] and college students [19], as well as the recommendation by the American Center for Disease Control and Prevention (CDC) to perform the test with straight elbow [25].

Espana-Romero et al. [27] explains the higher handgrip values produced by the TKK dynamometer in comparison with other dynamometers, partially by the fact that the grip span of this dynamometer is accurately adjusted to the adolescent's hand size by using the equations of Ruiz et al [18]. In our study we applied the equations adjusted for the hand size of children [15] when using the TKK dynamometer, and we also observed greater handgrip strength values in favour of the TKK vs the DynX dynamometer.

The greater difference between the recorded values with straight and bent arm, obtained with TKK vs DynX dynamometer in our study, might be explained by the influence of the dynamometer's weight and the greater distance of the tool from the hand grip, which is apparent especially for the TKK dynamometer. This might negatively influence the handgrip strength produced

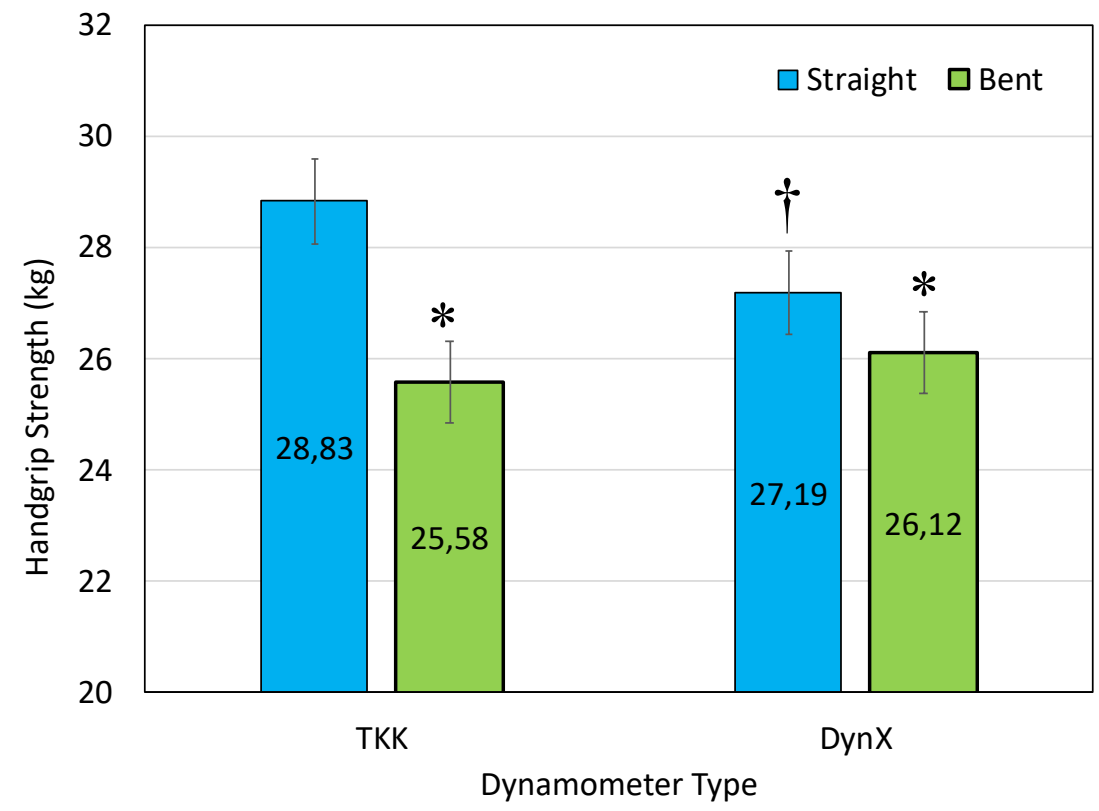

Figure 2. Comparison of handgrip strength measured with elbow at full extension (blue) and with elbow flexed at $90^{\circ}$ (green) in children aged 6 to 11 years $(n=60)$. Sum of right and left hand scores is shown, and was used for analyses. Data are expressed as mean \pm SE. $* p<0.001$ vs elbow at full extension. $\uparrow p<0.001$ vs TKK dynamometer with elbow at full extension. 

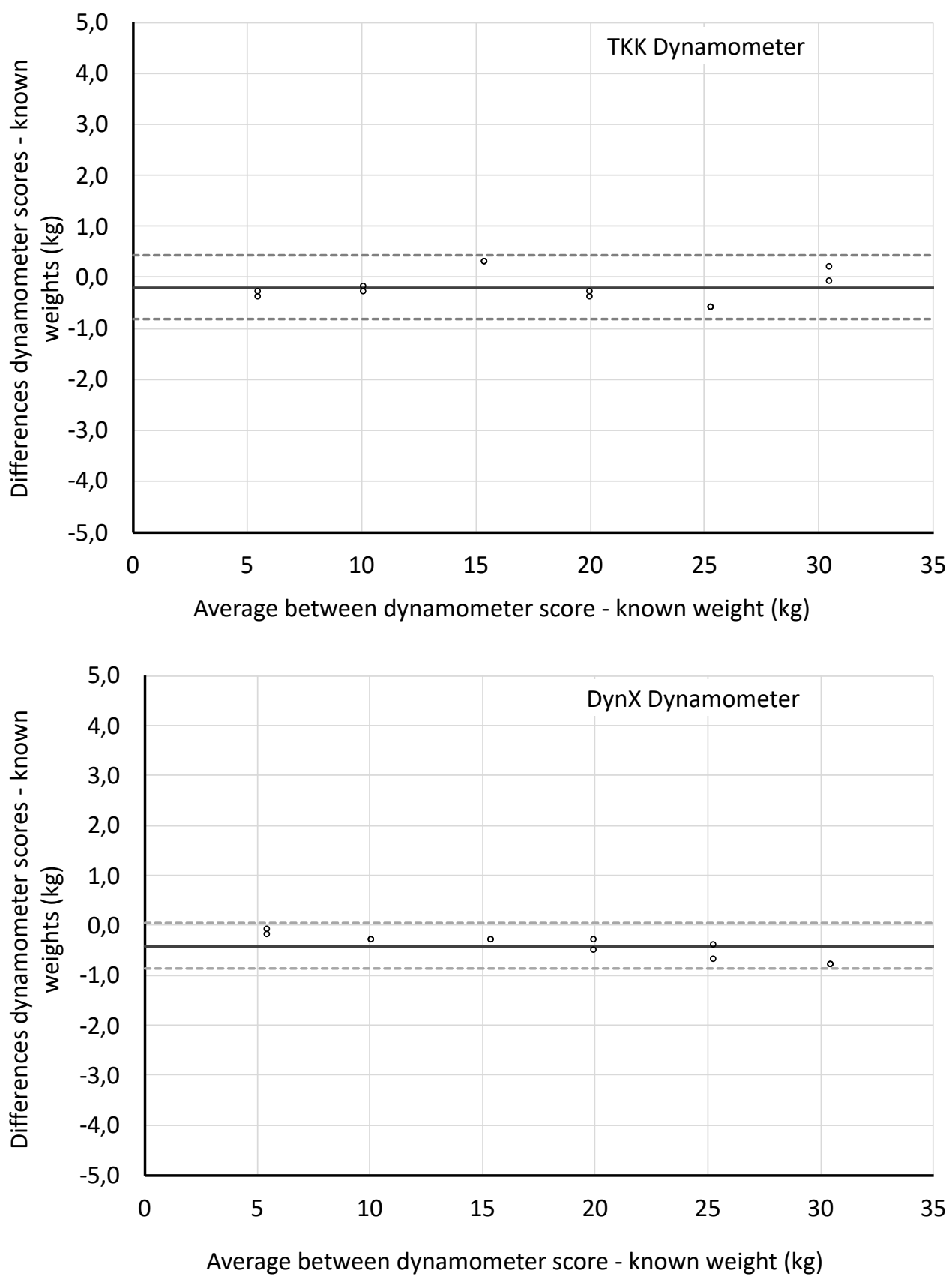

Figure 3. Validity between the TKK and DynX dynamometers and known weights by Bland and Altman plots [32]. The central line shows the mean difference (bias) between the dynamometer weight score and the known weight. The upper and lower lines represent $95 \%$ limits of agreement (mean difference \pm 1.96 SD of differences).

with bent elbow, specifically in children. This effect is smaller for the lighter dynamometer, DynX. When handgrip strength is assessed by using lighter (DynX) and shorter (Jamar) dynamometers in older individuals, such as adolescents [27] and young adults [26], no significant difference between the straight and bent elbow positions was reported.

Espana-Romero et al reported that the DynX dynamometer underestimates the handgrip strength levels $(-1.43 \mathrm{~kg}, \mathrm{p}<0.05)$, and the TKK dynamometer slightly overestimates handgrip strength $(0.49 \mathrm{~kg}, \mathrm{p}<0.05)$ when analysed vs known weights between the ranges of 5 to $70 \mathrm{~kg}$, but these systematic biases are more visible after reaching a handgrip strength above $40 \mathrm{~kg}$ [27]. In the present study, the validity analyses within the range of the children's possible handgrip strength $(5-30 \mathrm{~kg})$ suggested a systematic error for the DynX dynamometer $(-0.42 \mathrm{~kg}$, $\mathrm{p}<0.001$ ), but this underestimation is lower than the precision of an average analog dynamometer (i.e. $0.5 \mathrm{~kg}$ ). Our findings also suggested a non-significant systematic bias of $-0.20 \mathrm{~kg}(\mathrm{p}>0.05)$ for the TKK dynamometer, which is in agreement with Espana-Romero et al. within the range of 5-30 kg [27]. In contrast, the reported results in a recent study on validity of different models of TKK dynamometers ( 2 new digital, 1 old digital, 2 new analog and 1 old analog) vs known weights suggested that all of the TKK models underestimates the handgrip strength from $-0.94 \mathrm{~kg}$ to $-2.64 \mathrm{~kg}$ [37]. However, the authors 

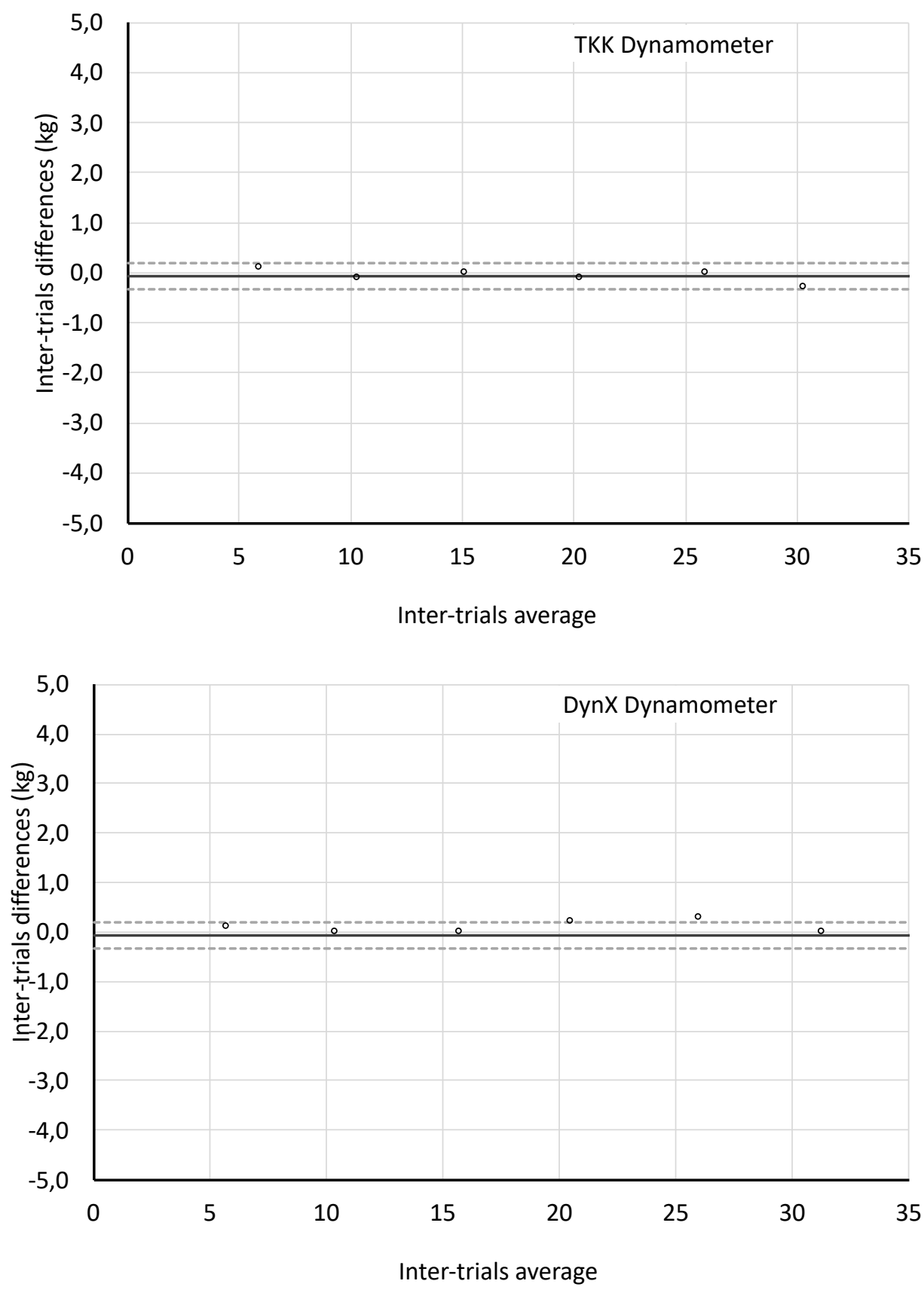

Figure 4. Reliability of weight trials of TKK and DynX dynamometers by Bland and Altman plots [32]. The central line shows the mean difference (bias) between the dynamometer weight score and the known weight. The upper and lower lines represent $95 \%$ limits of agreement (mean difference \pm 1.96 SD of differences).

believe that this underestimation could be due to the scale used to measure weights.

The reliability analyses in our study suggested a systematic bias of $-0.07 \mathrm{~kg}$ in the TKK, and $0.10 \mathrm{~kg}$ in the DynX dynamometer ( $p>0.05$ for both dynamometers), similar to those reported in other studies: $0.02 \mathrm{~kg}$ for the TKK and $0.26 \mathrm{~kg}$ for the DynX dynamometer ( $\mathrm{p}>$ $0.05)$ [27]; $-0.20 \mathrm{~kg}(\mathrm{p}>0.05)$ for the TKK dynamometer [36]. Overall, studies on test and retest of the TKK dynamometer showed consistency with a mean difference of less than $0.25 \mathrm{~kg}$ between trails [33-36]. However, when repeated measures are taken by using different TKK dynamometers (e.g., two digital dynamometers) or different models (e.g., digital vs analog), the systematic error is expected to range from 0.3 to $0.6 \mathrm{~kg}$ [37].

\section{Conclusions}

In conclusion, performing the handgrip strength test with elbow extended appears to be the most appropriate protocol in order to evaluate maximal handgrip strength in children. Both, TKK and DynX dynamometers provided sufficient results in terms of their reliability within the range of the children's handgrip strength, and the TKK dynamometer showed to be more valid.

\section{Conflict of interests}

The authors declare that there is no conflict of interests 


\section{References}

1. American College of Sports Medicine. ACSM's HealthRelated Physical Fitness Assessment Manual, Fourth Edition. Kaminsky L, editor. USA: Lippincott Williams and Wilkins; 2014.

2. Ruiz J, J. Castro-Pinero, E. Artero, F. Ortega, M. Sjostrom, J. Suni, et al. Predictive validity of health-related fitness in youth: a systematic review. Br J Sports Med. 2009;43(12):909-23. https://doi.org/10.1136/bjsm.2008.056499

3. Smith JJ, Eather N, Morgan PJ, Plotnikoff RC, Faigenbaum $\mathrm{AD}$, Lubans DR. The health benefits of muscular fitness for children and adolescents: a systematic review and meta-analysis. Sports Med. 2014;44(9):1209-23. https://doi.org/10.1007/s40279-014-0196-4

4. Ortega FB, Ruiz JR, Castillo MJ, Sjostrom M. Physical fitness in childhood and adolescence: a powerful marker of health. Int $J$ Obes (Lond). 2008;32(1):1-11. https://doi.org/10.1038/sj.ijo.0803774

5. Santos R, Mota J. The ALPHA health-related physical fitness test battery for children and adolescents. Nutr Hosp, 2011;26:1199-200. https://doi.org/10.1590/S0212-16112011000600001

6. Council of Europe. Recommendation no. r (87) 9 of the Committee of ministers to member states on the EUROFIT tests of physical fitness. [Internet]. 1987. [updated 2020 Jun 05; cited 2020 Jun 25]. Available from: http://www.coe.int/t/ dg4/epas/resources/sportpolicies_en.asp

7. Ruiz J, F. Ortega, A. Gutiérrez, D. Meusel, M. Sjöström, Castillo M. Health-related fitness assessment in childhood and adolescence: aEuropeanapproach based on theAVENA,EYHS and HELENA studies. $J$ Public Health. 2006;14(5):269-77. https://doi.org/10.1007/s10389-006-0059-z

8. Ortega FB, Cadenas-Sanchez C, Sanchez-Delgado G, Mora-Gonzalez J, Martinez-Tellez B, Artero EG, et al. Systematic review and proposal of a field-based physical fitness-test battery in preschool children: the PREFIT battery. Sports Med. 2015;45(4):533-55. https://doi.org/10.1007/s40279-014-0281-8

9. Bianco A, Jemni M, Thomas E, Patti A, Paoli A, Ramos Roque $\mathrm{J}$, et al. A systematic review to determine reliability and usefulness of the field-based test batteries for the assessment of physical fitness in adolescents - The ASSO Project. International Journal of Occupational Medicine and Environmental Health. 2015;28(3):445-78. https://doi.org/10.13075/ijomeh.1896.00393

10.Richards LG, Olson B, Palmiter-Thomas P. How forearm position affects grip strength. The American Journal of Occupational Therapy. 1996;50(2):133-8. https://doi.org/10.5014/ajot.50.2.133

11. Su CY, Lin JH, Chien TH, Cheng KF, Sung YT. Grip strength in different positions of elbow and shoulder. Archives of Physical Medicine and Rehabilitation. 1994;75(7):812-5. https://doi.org/10.1016/0003-9993(94)90142-2

12.Parvatikar VB, Mukkannavar PB. Comparative Study of Grip Strength in Different Positions of Shoulder and Elbow with Wrist in Neutral and Extension Positions. Journal of Exercise Science and Physiotherapy. 2009;5(2):67-75.

13. Watanabe T, Owashi K, Kanauchi Y, Mura N, Takahara M, Ogino T. The short-term reliability of grip strength measurement and the effects of posture and grip span. The Journal of Hand Surgery. 2005;30(3):603-9. https://doi.org/10.1016/j.jhsa.2004.12.007

14.El-Sais W, Mohammad W. Influence of different testing postures on hand grip strength. European Scientific Journal. 2014;10(36):290-301.

15.Espana-Romero V, Artero EG, Santaliestra-Pasias AM, Gutierrez A, Castillo MJ, Ruiz JR. Hand span influences optimal grip span in boys and girls aged 6 to 12 years. The Journal of Hand Surgery. 2008;33(3):378-84. https://doi.org/10.1016/j.jhsa.2007.11.013

16.Harkonen R, Piirtomaa M, Alaranta H. Grip strength and hand position of the dynamometer in 204 Finnish adults. Journal of Hand Surgery. 1993;18(1):129-32. https://doi.org/10.1016/0266-7681(93)90212-X

17.Ruiz-Ruiz J, Mesa JL, Gutierrez A, Castillo MJ. Hand size influences optimal grip span in women but not in men. The Journal of Hand Surgery. 2002;27(5):897-901. https://doi.org/10.1053/jhsu.2002.34315

18.Ruiz JR, Espana-Romero V, Ortega FB, Sjostrom M, Castillo MJ, Gutierrez A. Hand span influences optimal grip span in male and female teenagers. The Journal of Hand Surgery. 2006;31(8):1367-72. https://doi.org/10.1016/j.jhsa.2006.06.014

19.Balogun JA, Akomolafe CT, Amusa LO. Grip strength: effects of testing posture and elbow position. Archives of Physical Medicine and Rehabilitation. 1991;72(5):280-3.

20.Desrosiers J, Bravo G, Hebert R, Mercier L. Impact of elbow position on grip strength of elderly men. Journal of Hand Therapy, 1995;8(1):27-30. https://doi.org/10.1016/S0894-1130(12)80153-0

21.Kuzala EA, Vargo MC. The relationship between elbow position and grip strength. The American Journal of Occupational Therapy, 1992;46(6):509-12. https://doi.org/10.5014/ajot.46.6.509

22. Mathiowetz V, Rennells C, Donahoe L. Effect of elbow position on grip and key pinch strength. The Journal of Hand Surgery. 1985;10(5):694-7. https://doi.org/10.1016/S0363-5023(85)80210-0

23.Oxford KL. Elbow positioning for maximum grip performance. Journal of Hand Therapy, 2000;13(1):33-6. https://doi.org/10.1016/S0894-1130(00)80050-2

24.Fess E, Moran C. Clinical Assessment Recommendations. USA: American Society of Hand Therapists; 1981.

25.NHANES. Muscle Strength Procedures Manual. National Health and Nutrition Examination Survey (NHANES); 2013.

26.Kattel BP, Fredericks TK, Fernandez JE, Lee DC. The effect of upper-extremity posture on maximum grip strength. International Journal of Industrial Ergonomics. 1996;18(5-6):423-9. https://doi.org/10.1016/0169-8141(95)00105-0

27.España-Romero V, Ortega FB, Vicente-Rodríguez G, Artero EG, Rey JP, Ruiz JR. Elbow Position Affects Handgrip Strength in Adolescents: Validity and Reliability of Jamar, DynEx, and TKK Dynamometers. Journal of Strength and Conditioning Research, 2010;24:272-7. https://doi.org/10.1519/JSC.0b013e3181b296a5

28. Shechtman O, Gestewitz L, Kimble C. Reliability and validity of the DynEx dynamometer. Journal of Hand Therapy, 2005;18(3):339-47. https://doi.org/10.1197/j.jht.2005.04.002

29.van den Beld WA, van der Sanden GA, Sengers RC, Verbeek AL, Gabreels FJ. Validity and reproducibility of the Jamar dynamometer in children aged 4-11 years. Disability and rehabilitation. 2006;28(21):1303-9. https://doi.org/10.1080/09638280600631047

30.Chatburn RL. Evaluation of instrument error and method agreement. AANA journal. 1996;64(3):261-8.

31.Atkinson G, Nevill AM. Statistical methods for assessing measurement error (reliability) in variables relevant to sports medicine. Sports Med. 1998;26(4):217-38. https://doi.org/10.2165/00007256-199826040-00002

32.Bland J, Altman D. Statistical methods for assessing agreement between two methods of clinical measurement. Lancet. 1986;1:307-10. https://doi.org/10.1016/S0140-6736(86)90837-8 
33.Carbonell-Baeza A, Alvarez-Gallardo IC, Segura-Jimenez V, Castro-Pinero J, Ruiz JR, Delgado-Fernandez M, et al. Reliability and feasibility of physical fitness tests in female fibromyalgia patients. Int J Sports Med. 2015;36(2):157-62. https://doi.org/10.1055/s-0034-1390497

34.España-Romero V, Artero EG, Jimenez-Pavón D, Cuenca-Garcia $M$, Ortega FB, Castro-Piñero $J$, et al. Assessing Health-Related Fitness Tests in the School Setting: Reliability, Feasibility and Safety; The ALPHA Study. Int J Sports Med, 2010;31:490-7. https://doi.org/10.1055/s-0030-1251990

35.Cadenas-Sanchez C, Martinez-Tellez B, SanchezDelgado G, Mora-Gonzalez J, Castro-Pinero J, Lof M, et al. Assessing physical fitness in preschool children: Feasibility, reliability and practical recommendations for the PREFIT battery. J Sci Med Sport. 2016;19(11):910-5. https://doi.org/10.1016/j.jsams.2016.02.003

36.Fernandez Santos JR, Ruiz JR, Gonzalez-Montesinos JL, Castro-Pinero J. Reliability and Validity of Field-Based Tests to Assess Upper-Body Muscular Strength in Children Aged 6-12 Years. Pediatr Exerc Sci. 2016;28(2):331-40. https://doi.org/10.1123/pes.2015-0196

37.Cadenas-Sanchez C, Sanchez-Delgado G, MartinezTellez B, Mora-Gonzalez J, Lof M, Espana-Romero $\mathrm{V}$, et al. Reliability and Validity of Different Models of TKK Hand Dynamometers. The American Journal of Occupational Therapy, 2016;70(4):7004300010. https://doi.org/10.5014/ajot.2016.019117

38.MDSystems.Inc., inventorDynX - Electronic Hand Dynamometer \& Programmable Hand Exerciser, User's Guide. USA; 2014.

39. Cohen J. Statistical Power Analysis for the Behavioral Sciences. Edition S, editor. USA: Lawrence Erlbaum Associates; 1988.

40.Sawilowsky S. New Effect Size Rules of Thumb. Journal of Modern Applied Statistical Methods. 2009;8(2):597-9. https://doi.org/10.22237/jmasm/1257035100

41.Kolimechkov S, Petrov L, Alexandrova A. Alpha-fit test battery norms for children and adolescents from 5 to 18 years of age obtained by a linear interpolation of existing European physical fitness references. European Journal of Physical Education and Sport Science. 2019;5(4):1-14.

42.Council of Europe. Testing physical fitness - Eurofit, provisional handbook. 1983.

43.Kolimechkov S. Physical Fitness Assessment in Children and Adolescents: A Systematic Review. European Journal of Physical Education and Sport Science. 2017;3(4):65-78.

44.Bianco A, Mammina C, Jemni M, Filippi AR, Patti A, Thomas E, et al. A Fitness Index model for Italian adolescents living in Southern Italy: the ASSO project. J Sports Med Phys Fitness. 2016;56(11):1279-88.

45.Ahrens W, Bammann K, de Henauw S, Halford J, Palou A, Pigeot I, et al. Understanding and preventing childhood obesity and related disorders-IDEFICS: A European multilevel epidemiological approach. Nutrition, Metabolism and Cardiovascular Diseases, 2006;16:302-8. https://doi.org/10.1016/j.numecd.2006.01.011

46.Ortega F, E. Artero, J. Ruiz, V. España-Romero, D. Jiménez-Pavón, G. Vicente-Rodriguez, et al. Physical fitness levels among European adolescents: the HELENA study. British Journal of Sports Medicine. 2011;45:20-9. https://doi.org/10.1136/bjsm.2009.062679

47.Cronin J, Lawton T, Harris N, Kilding A, McMaster DT.ABrief Review ofHandgripStrengthandSportPerformance.Journalof Strength and Conditioning Research. 2017;31(11):3187-217. https://doi.org/10.1519/JSC.0000000000002149

48.Bonitch-Gongora JG, Bonitch-Dominguez JG, Padial P, Feriche B. The effect of lactate concentration on the handgrip strength during judo bouts. Journal of Strength and Conditioning Research. 2012;26(7):1863-71. https://doi.org/10.1519/JSC.0b013e318238ebac

\section{Information about the authors:}

Stefan Kolimechkov; (Corresponding author); http://orcid.org/0000-0003-0112-2387; dr.stefan.kolimechkov@gmail.com; STK SPORT; London, United Kingdom.

Jose Castro-Piñero; https://orcid.org/0000-0002-7353-0382; jose.castro@uca.es; Department of Physical Education, Faculty of Education Sciences, University of Cadiz, Puerto Real, Spain.

Lubomir Petrov; https://orcid.org/0000-0003-1209-959X; dr.lubomir.petrov@gmail.com; Department of Physiology and Biochemistry, National Sports Academy, Sofia, Bulgaria.

Albena Alexandrova; https://orcid.org/0000-0002-7007-3665; a_alexandrova_bas@yahoo.com; Department of Physiology and Biochemistry, National Sports Academy, Sofia, Bulgaria.

Cite this article as:

Kolimechkov S, Castro-Piñero J, Petrov L, Alexandrova A. The effect of elbow position on the handgrip strength test in children: validity and reliability of TKK 5101 and DynX dynamometers. Pedagogy of physical culture and sports, 2020;24(5):240-247.

https://doi.org/10.15561/26649837.2020.0504

This is an Open Access article distributed under the terms of the Creative Commons Attribution License, which permits unrestricted use, distribution, and reproduction in any medium, provided the original work is properly cited (http://creativecommons.org/licenses/by/4.0/deed.en).

Received: 20.02 .2020

Accepted: 27.03.2020; Published: 30.10 .2020 